\title{
Lorentz Invariance Violation Tests in Astroparticle Physics
}

\author{
Humberto Martínez-Huerta *(D), Rodrigo Guedes Lang (D) and Vitor de Souza (D) \\ Instituto de Física de São Carlos, Universidade de São Paulo, São Carlos 13566-590, SP, Brazil; \\ rodrigo.lang@usp.br (R.G.L.); vitor@ifsc.usp.br (V.d.S.) \\ * Correspondence: humbertomh@ifsc.usp.br
}

Received: 13 May 2020; Accepted: 9 June 2020; Published: 27 July 2020

\begin{abstract}
In this review, we present the latest exclusion limits obtained from astroparticles on Lorentz Invariance Violation (LIV) in the photon sector. We discuss the techniques known as energy-dependent time delay or time lag, subluminal pair production threshold shift, suppression of air shower formation, superluminal photon decay, and superluminal photon splitting. Perspectives for future results on LIV with the next generation of experiments are also addressed.
\end{abstract}

Keywords: Lorentz invariance violation; gamma rays; cosmic rays

\section{Introduction}

Lorentz symmetry has a fundamental role in the physics of elementary particles and relativity. Exploring its validity limits has been a significant motive for experimental and theoretical studies, and furthermore, by also exploring a symmetry violation [1,2]. On the other hand, the formulation of a quantum theory of gravity, which is one of the main challenges in physics, is able to give rise to some Lorentz invariance violation (LIV) [3-5].

LIV phenomena in the photon sector can lead to novel measurable effects such as photon decays, vacuum Cherenkov radiation, the energy-dependent speed of light, and also to the modifications of well-known processes, like the electron-position pair production by photons, and the suppression in air shower formation [6-10]. These effects predict very singular imprints in astrophysical observations because of the high energies and the large propagation distances [11]. Recently, the community has shown renewed interest in LIV tests with astroparticles due to the high precision of the new measurements. In this review, we present a summary of such findings and prospects for the current and future generations of gamma ray telescopes and ultra-high-energy cosmic ray (UHECR) observatories.

In Section 2, we discuss the phenomenological approach to the LIV; then, in Section 3, we address the LIV energy-dependent time delay. In Section 4, we review the LIV implications on the electron-positron pair $\left(\mathrm{e}^{+} \mathrm{e}^{-}\right)$production due to a high energy photon interacting, in its propagation from distant sources to the Earth, with a photon from an extra-galactic background light (EBL) or cosmic microwave background (CMB). Next, in Sections 5 and 6, we discuss the photon decay and photon splitting processes. Later, in Section 7, we review the suppression in the formation of atmospheric air showers. Finally, in Section 8, we present a summary table and figures with the most robust exclusion limits to LIV due to the lack of such signatures in astrophysical data and discuss the systematics and possible future of each technique.

\section{Modified Dispersion Relation for Astroparticle Tests}

In the Lorentz invariant (LI) standard model of particles (SM), the spontaneous symmetry breaking of the Lorentz symmetry [1,12], or the introduction of an explicit Lorentz-violating term in the SM Lagrangian [13], is able to induce modifications on the dispersion relation (MDR) for particles. From a 
phenomenological approach, a generalization of these LIV effects converges on the introduction of a general function of the energy and momentum [11]. There are many and diverse forms of MDR for different particles and underlying LIV-theories; however, some of them may steer to phenomenology alike. This has been proven to be advantageous when testing LIV in extreme environments like in astroparticle physics [14]. Therefore, it is convenient to address a family of MDRs, which can be described by the expression (Hereafter, we use natural units, $c=\hbar=1$, unless other units are explicitly given.)

$$
E_{a}^{2}-p_{a}^{2}=m_{a}^{2} \pm\left|\delta_{a, n}\right| p_{a}^{n+2},
$$

where $a$ represents the particle type, $E_{a}$ is its energy, $p_{a}$ is its the momentum, and $m_{a}$ is its mass. The leading order of the modification by the underlying theory is $n$, and $\delta_{a, n}$ stands for a LIV parameter. In some effective field theories, $\delta_{a, n}=\epsilon_{a}^{(n)} / M$ is used, where $\epsilon_{a}^{(n)}$ are the LIV coefficients, and $M$ is the energy scale of the new physics, such as the energy of the Plank scale, $E_{\mathrm{Pl}} \approx 1.22 \times 10^{28} \mathrm{eV}$, or some Quantum Gravity energy scale, $E_{\mathrm{QG}}$. The sign \pm stands for the so-called superluminal $(+)$ or subluminal $(-)$ dominant phenomena, relative to the light speed in a LI-vacuum, $c$. For massless particles such as photons $\left(m_{a}=0\right)$, and approximately for high-energy astroparticles $\left(p_{a}, E_{a} \gg m_{a}\right.$ and $\left.p_{a} \approx E_{a}\right)$, to the first order in $\delta_{a, n}$ Equation (1) becomes

$$
E_{\gamma}^{2}-p_{\gamma}^{2}= \pm\left|\delta_{\gamma, n}\right| E_{\gamma}^{n+2}
$$

For simplicity, when $n>0$, we write the LIV parameter in terms of a LIV energy scale, $E_{L I V}^{(n)}=\left(\left|\delta_{\gamma, n}\right|\right)^{-1 / n}$.

\section{Energy-Dependent Time Delay}

In a Lorentz invariant regime, photons propagate in vacuum at the same speed independently of their energy, $E_{\gamma}$. However, the modified dispersion relation given in Equation (2) has the consequence that photons with different energies propagate with different speeds through the LIV vacuum [15-17]. The LIV effect forecasts that photons emitted from an astrophysical source, such as an active galactic nucleus (AGN), a gamma ray burst (GRB), or a blazar, should arrive at different times [5,17-19]. The time difference including cosmological corrections is given by

$$
\Delta t=\frac{1+n}{2 H_{0}} \frac{\Delta E^{n}}{\left(E_{\mathrm{LIV}}^{(n)}\right)^{n}} \int_{0}^{z} \frac{(1+z)^{n} \mathrm{~d} z}{h(z)}, \quad h(z)=\sqrt{\Omega_{m}(1+z)^{3}+\Omega_{\Lambda}},
$$

where $H_{0}$ is the Hubble constant at the present time, $z$ is the distance between the source and the Earth, and $\Delta E^{n}$ is the photon energy difference, $E_{\gamma_{2}}^{n}-E_{\gamma_{1}}^{n}$, where $E_{\gamma_{2}}>E_{\gamma_{1}}$. The distance element in an expanding universe is given by $h(z)$, where $\Omega_{m}$ and $\Omega_{\Lambda}$ are the LI cosmological parameters in $\Lambda C D M$ Cosmology evaluated today [20]. The authors of [15,21] reported earlier studies in this type of test, and the authors of [18] extended it to include corrections due to the expansion to the universe, leading to Equation (3). Nevertheless, there is also a cosmological model independent approach, see for instance in [22,23].

Strong superluminal and subluminal exclusion limits to the Lorentz violation coefficients with $n=1$ and 2 were published using the observations of the GRB090510 in [24], while recent limits using the Crab Nebula are reported in [25-27] and the blazar Mrk 501 in [28]. The authors of [21,29-31] present some previous limits studying GRBs. For comparison, we show strong and recent LIV limits in Table 1 and Figure 1.

Although this is one of the most common techniques, it depends largely on a good observation of very energetic and unpredictable events, such as GRBs. Moreover, it is common in these studies to assume that the observed photons were emitted simultaneously from the source and the effects of LIV meaningfully dominate the time delay. Recent LIV studies focusing on AGNs [32] and GRBs (see for instance in [33] and references therein) point that the intrinsic time delay of the source is significant and 
needs to be considered in this type of LIV analysis, so that more detailed studies should be developed in the near future.

The Large High Altitude Air Shower Observatory (LHAASO) projects to study the Universe at very high energies in cosmic and gamma rays. LHAASO will be sensitive at energies on the range of 100-1000 TeV. It will include a surface water Cherenkov detector array (LHAASO/WCDA), which will cover a total area of $\sim 78,000 \mathrm{~m}^{2}$ and incorporate 3120 detector units. By testing GRBs with the expected performance of the LHAASO/WCDA, reference sensitivity limits to LIV for $n=1$ and 2 were reported in [34]. We also show these sensitivity limits in Table 1 and Figure 1.
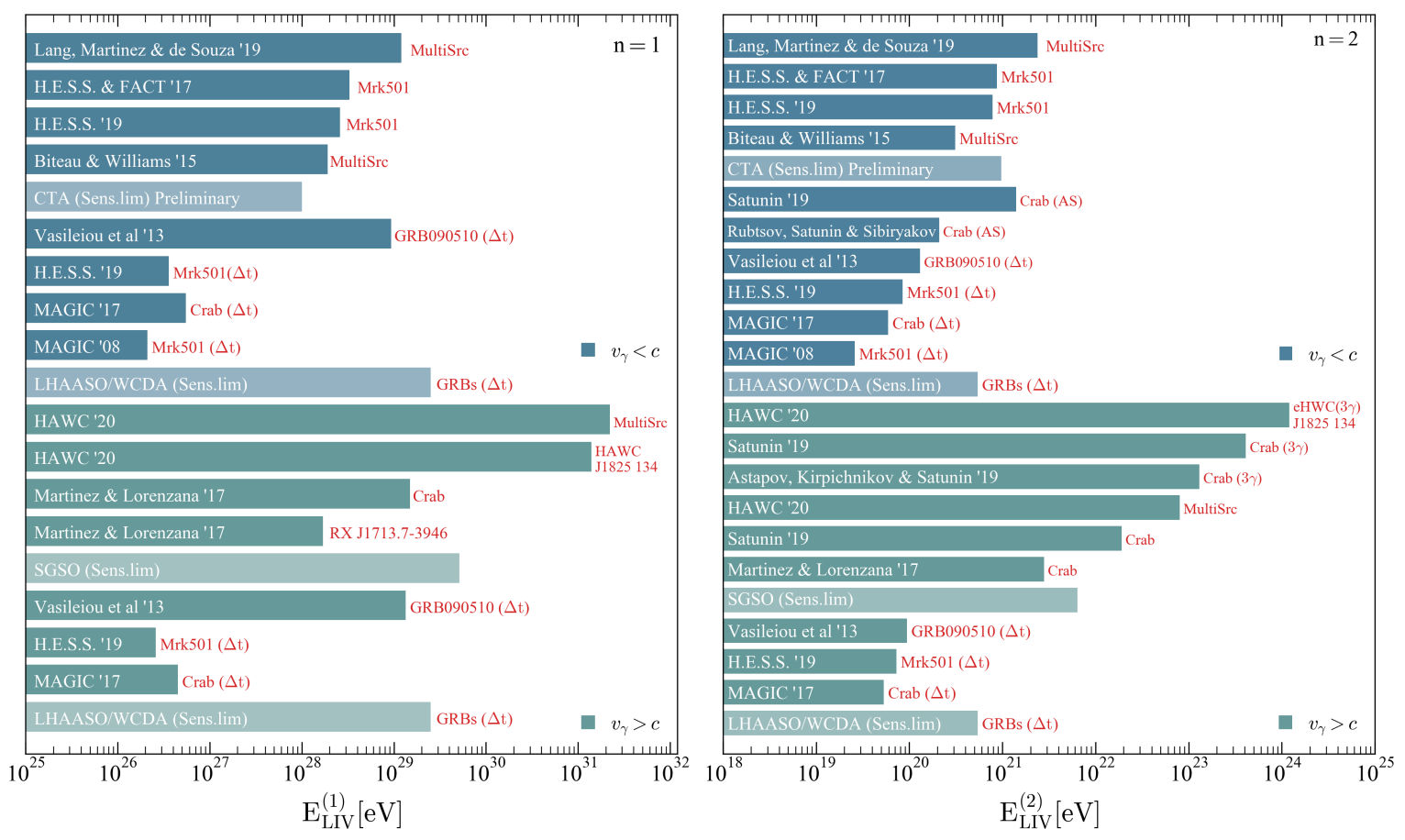

Figure 1. Comparison of strong and recent exclusion bounds on LIV coming from the lack of signatures of energy-dependent time delay, photon decay, photon splitting, suppression of air shower formations, and pair-production threshold shifts in astrophysical data. At the top (blue, $v_{\gamma}<c$ ), there are subluminal limits and at the bottom (green, $v_{\gamma}>c$ ) superluminal ones. The left and right panels are for the approximation orders $n=1$ and 2, respectively. See Table 1 for further information about these limits.

\section{Pair-Production Threshold Shift}

Gamma rays $(\gamma)$ propagating from distant sources to Earth interact with the background photons $\left(\gamma_{b}\right)$ being able to produce $\mathrm{e}^{+} \mathrm{e}^{-}$, through the process of pair production, $\gamma \gamma_{\mathrm{b}} \rightarrow \mathrm{e}^{+} \mathrm{e}^{-}$. This has the effect of a significant energy attenuation in the gamma ray energy, $E_{\gamma}$ [35]. The minimum energy that a $\gamma_{\mathrm{b}}$ needs to produce $\mathrm{a} \mathrm{e}^{+} \mathrm{e}^{-}$, for head-on collisions, is given by $m_{\mathrm{e}}^{2} / E_{\gamma}$. However, when LIV is considered [36-39], the threshold energy is shifted to

$$
E_{\gamma_{\mathrm{b}}}^{\text {th }}=\frac{m_{\mathrm{e}}^{2}}{E_{\gamma}}-\frac{1}{4} \delta_{\gamma, n} E_{\gamma}^{n+1}
$$

In the expression above, subluminal LIV yields an increase in the energy threshold of the interaction, while superluminal leads to a decrease. The cumulative outcome of this phenomenon may result in measurable variations in the expected attenuation of the gamma rays flux, derived from the gamma ray interaction with the $\gamma_{\mathrm{b}}[7,40,41]$.

Equation (4) considers LIV only in photons; however, if LIV is also included for electrons and positrons, $\delta_{\gamma, n} \rightarrow \delta_{n}^{\text {tot }}$, where $\delta_{n}^{\text {tot }}$ is a linear combination of the LIV coefficients of the electrons, 
positrons, and photons, so that the LIV phenomenology derived in both cases is preserved. For further discussion, see in [42] and references within.

The optical depth involving the LIV effects is found using the following expression (see for instance in [40] and references therein),

$$
\tau_{\gamma}\left(z, \theta, \eta_{\mathrm{b}}, E_{\gamma} ; n, \delta_{n}^{\text {tot }}\right)=\int_{0}^{z} \mathrm{~d} z \frac{c}{H_{0}(1+z) h(z)} \int_{-1}^{1} \mathrm{~d}(\cos \theta) \frac{1-\cos \theta}{2} \int_{E_{\gamma_{\mathrm{b}}}^{\mathrm{th}}}^{\infty} \mathrm{d} E_{\gamma_{\mathrm{b}}} \eta_{\mathrm{b}}\left(E_{\gamma_{\mathrm{b}}}, z\right) \sigma\left(E_{\gamma}, E_{\gamma_{\mathrm{b}}}, z\right),
$$

where $\eta_{\mathrm{b}}$ is the density of $\gamma_{\mathrm{b}}$ and the cross section of the pair production process is $\sigma$, which it is a function of the gamma ray energy, $E_{\gamma}$, the background photon energy $E_{\gamma_{b}}$, and $z$, the distance between the source and the Earth [43]. The angle between particles is represented by $\theta$, which ranges between $[-\pi,+\pi]$. As in the section above, $h(z)$ is once again the distance element in an expanding universe, and $c$ is the speed of light in a LI-vacuum. Finally, $E_{\gamma_{b}}^{\text {th }}$ is the background photon energy threshold as given by Equation (4).

Using Equation (5), the resulting mean-free path is given by

$$
\lambda=\frac{c z}{H_{0} \tau_{\gamma}} \approx 4[\mathrm{Gpc}] \times \frac{z}{\tau_{\gamma}},
$$

which significantly changes when LIV is considered, such that, if LIV is subluminal $\lambda_{\mathrm{LI}} / \lambda_{\mathrm{LIV}}<1$, while it is $>1$ when LIV photons are superluminal. That is, subluminal LIV predicts more photons arriving from further distances and sources, while superluminal will significantly reduce the expected gamma rays from distant sources [44,45].

In the $\mathrm{TeV}$ gamma ray energy range, the production of $\mathrm{e}^{+} \mathrm{e}^{-}$is dominated by the interaction with the EBL photons. The cumulative effect of these phenomena forecasts modifications in a source spectra. As an example, Figure 2 shows the gamma ray attenuation

$$
a\left(E_{\gamma}, z ; \delta_{n}, n\right)=\exp (-\tau)
$$

for two different LIV scenarios when $n=1$, and for a value of $E_{\mathrm{LIV}}^{(1)}$ of the order of magnitude of current exclusion limits [41]. For these LIV scales, effects on the spectrum above a few tens of $\mathrm{TeV}$ are expected. The subluminal effect predicts a recovery in the spectra at the highest energies, while the superluminal case has the effect of reducing the gamma ray flux. In this example, we use Dominguez et al.'s (2011) EBL model [46], although using other EBL models, such as those in [47,48], results in similar effects [41].

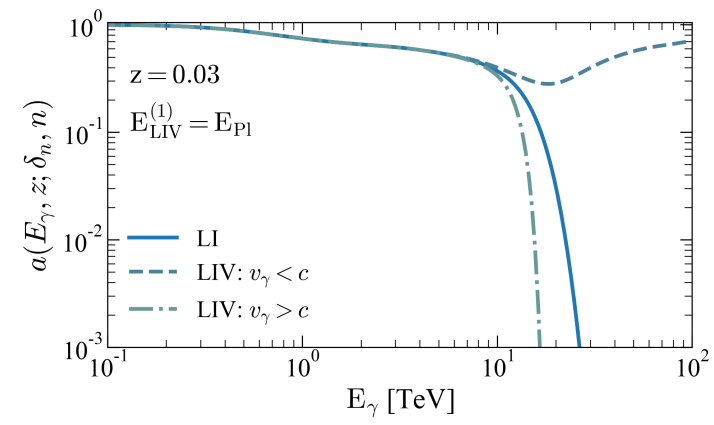

(a)

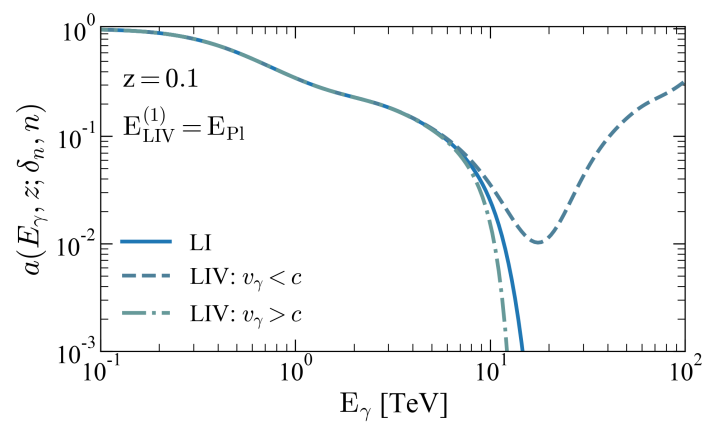

(b)

Figure 2. Gamma ray absorption for LI and LIV scenarios. When LIV is subluminal, there is a recovery in the flux while the superluminal scenario has the effect to reduce the gamma ray flux. The effect depends on the LIV energy scale, the gamma ray energy and the redshift to the source, as it can be compared between panels $(\mathbf{a}, \mathbf{b})$ for different red shifts but the same LIV value.

Earlier tests of this method were reported in [49,50] by looking at Mrk 501 and Mrk 421, and assuming a drop-off above a few $\mathrm{TeV}$ due to intergalactic absorption. A significant improvement was presented later in [7], by testing a large collection of $\mathrm{TeV}$ spectra from blazars and considering 
uncertainties on the EBL. Although they found no significant evidence for anomalies, LIV constraints are reported to the linear and quadratic LIV terms. The authors of [28] use the observations of Mrk 501 from H.E.S.S. to improve the LIV exclusion limits. The authors of [41] develop a new analysis procedure to improve the statistical test using an updated $\mathrm{TeV}$ gamma ray data set. Once again, the authors of [41] find no LIV signatures, and thus stringent exclusion limits are reported with this method. These limits are robust under several tested systematic uncertainties.

As for sensitivity limits, the Cherenkov Telescope Array (CTA) reported prospects to test LIV through the photo pair production threshold shifts. CTA will be capable of detecting gamma rays with energies ranging from about $20 \mathrm{GeV}$ to more than $300 \mathrm{TeV}$ with unprecedented precision both in energy and directional reconstruction [51]. This will cause an unmatched opportunity for this and other LIV tests [52]. By performing and studying the simulations of the CTA observations of the nearby blazar Mrk501 and 1ES 200+209 spectra, CTA shows the preliminary outcome that it will be sensitive to the subliminal pair production threshold shift effects produced at least up to the $E_{\mathrm{Pl}}$ for the linear LIV coefficient $[53,54]$.

The limits discussed in this section, including the preliminary sensitivity limits, are presented in Table 1 and Figure 1 and compared with the limits coming from the other LIV effects presented in this review.

As for the TeV gamma ray energy window, the evolution line of this method is now well established, and it will grow with the arrival of further astrophysical data taken by the current and the next gamma ray telescopes.

Ultra-high-energy photons, on the other hand, provide a different challenge for this method, since no UHE photon events have been observed so far [55]; in the $10^{14.5} \mathrm{eV}<E_{\gamma}<10^{19} \mathrm{eV}$ photon energy range, the photo-production of electron-positron pairs is dominated by the interaction with CMB photons, and at the highest end of the spectrum, $E_{\gamma}>10^{19} \mathrm{eV}$, the interaction is dominated by photons from the Radio Background. In order to face the challenge, the authors of $[39,40,56]$ performed a search for LIV signatures in the propagation of secondary UHE-photons emitted on the propagation of ultra-high-energy cosmic rays (UHECR), by computing the ultra-high-energy photon flux on Earth, the so-called GZK-photon flux due to the cosmic ray energy limit deduced by Kenneth Greisen, Vadim Kuzmin, and Georgiy Zatsepin (GZK) [57,58]. In addition, the authors of [40] also innovate by considering by first time, different UHECR source distribution, and injection models in this line of studies. Among them, there is the combination of models that has presented the best description of the UHECR composition data and energy spectrum, which considers a mixed composition of different primary cosmic rays nuclei and a source distribution that follows a GRB rate evolution [59].

The resulting subluminal LIV effect is the increase in the predicted GZK-photon flux. It is possible to find the maximum increase from these effects, by taking the limit $\delta_{n} \rightarrow-\infty$ (for $n=1$ and 2, the limit is equivalent to $\left.E_{\mathrm{LIV}}^{(n)} \rightarrow 0\right)[40,42]$. When confronting this outcome, in the astrophysical scenario that better describes UHECR data, with the most updated upper bounds on the integrated photon flux imposed by the Pierre Auger Observatory [55], there are already LIV-scenarios that predicts a GZK-photons flux above these upper limits; thus they are excluded (for a wider discussion on this matter, please review [42] and the references within). We also show these limits in Table 1.

Recent works have obtained similar limits with other astrophysical scenarios [60]. These rejection limits are many orders of magnitude more stringent than those established through $\mathrm{TeV}$ gamma rays. They strongly rely, however, on astrophysical assumptions about the UHECRs propagation and sources, such as the injected spectra and composition and the redshift evolution. Little is known about the sources of UHECR, and while different models can properly describe the spectral and composition data, the LIV limits obtained with each of these models can differ by several orders of magnitude, as discussed in [40]. Therefore, the comparison from results obtained with UHECR and gamma rays is not straightforward, given the different assumptions, energies, and systematics involved.

The unique subluminal signature reviewed in this section is quite attractive for LIV tests using gamma ray spectra and UHE photon limits, however not very promising for the superluminal cases. 
As it can be seen in the Figure 2, distinguishing between the intrinsic source effects due to LI absorption and superluminal LIV effects may not be trivial and therefore it requires some new techniques and more significant characterization of LIV effects at a level that is currently below the precision of current astrophysical telescopes and models. Besides, as it will be seen in the next sections, there are other more promising channels for superluminal LIV with renewed interest in the community.

\section{Photon Decay}

There are processes forbidden in the LI physics but permitted under LIV which can lead to restrictive scenarios for astroparticles, such as the photon decay. This process is described by $\gamma \rightarrow \ell^{+} \ell^{-}$, where $\ell$ stand for any charged particle; however, the lightest and most common channel belongs to $\gamma \rightarrow \mathrm{e}^{+} \mathrm{e}^{-}$, which is the one we consider below.

Photon decay to an $\mathrm{e}^{+} \mathrm{e}^{-}$pair has been studied in the context of MDR and effective field theories like the Standard Model Extension (see for instance in $[6,8,61]$ ). This process has an energy threshold, which for $E_{\gamma} \gg m_{\mathrm{e}}$ and for any order $n$ is

$$
E_{\gamma}^{\mathrm{th}} \sim\left(\frac{4 m_{\mathrm{e}}^{2}}{\delta_{\gamma, n}}\right)^{1 /(n+2)}
$$

Below this threshold, photons behave as in the LI regime and do not decay. Moreover, if $\delta_{\gamma, n} \rightarrow 0$, then $E_{\gamma}^{\text {th }} \rightarrow \infty$. The modified decay rates for the photon decay towards an $\mathrm{e}^{+} \mathrm{e}^{-}$steadily grow with $E_{\gamma}$, so that, once this process is allowed, it is very fast and effective $[6,8,13,61]$. Photon decay sharply restricts the possible propagation of LIV photons to extremely short distances, on the order of centimeters or shorter; therefore, it is improbable that a LIV photon propagates through astronomical distances, and a direct test of superluminal LIV rises from any high energy cosmic photon observation. Then, handling Equation (8), the explicit limit is, $\delta_{\gamma, n} \lesssim 4 m_{\mathrm{e}}^{2} / E_{\gamma}^{n+2}$.

The authors of [13] reported early exclusion limits for the corresponding $n=0$ scenario, by considering $\sim 20 \mathrm{TeV}$ photons from the Crab Nebula, while the authors of [49] consider $\sim 20 \mathrm{TeV}$ observed by CANGAROO [62]. The advent of new data by HEGRA [63], and the observations of the SNR RX J1713.7-394 by H.E.S.S. [64], allowed more restrictive limits due to the absence of photon decay $[6,8,65]$. Tevatron photons have also been used to test photon decay [61]; however, due to the lower photon energies involved when compared with astrophysical photon observations, constraints on photon decay are less restrictive. At very high photon energies, the strong effect of photon decay forecasts a hard cutoff in the shape of the astrophysical spectra of a given source. The High Altitude Water Cherenkov (HAWC) observatory using its most recent findings of gamma ray observation with photon energies above $100 \mathrm{TeV}$ [66,67], jointly with a dedicated search of such hard cutoff in different spectra, results in the stringent constraints combining the Crab and three other sources [68-70].

The next generation of wide field-of-view gamma ray survey instruments, such as the Southern Gamma-Ray Survey Observatory (SGSO) and the Southern Wide-field Gamma ray Observatory (SWGO), will be sensitive to gamma rays with energies from $100 \mathrm{GeV}$ to hundreds of TeV. This is ideal for extending the search of photon decay signatures in the Southern Hemisphere. So that, in their science motivation papers [71,72], there are LIV sensitivity exclusion limits through the observation of the SNR RX J1713.7-394 and the absence of photon decay. We include these limits and those addressed in this section in Table 1 and Figure 1. 
Table 1. Strong and recent astrophysical LIV limits. $\left|\delta_{0}\right|$ are upper limits meanwhile $E_{\mathrm{LIV}}^{(n)}$ are lower limits. PP stands for pair production, $\Delta t$ for energy-dependent time delay, AS for suppression of air shower formation, $(3 \gamma)$ for photons splitting, and PD for photon decay. In addition, (+) indicates $\delta_{\gamma, n}>0$, while (-) is for $\delta_{\gamma, n}<0$.

\begin{tabular}{|c|c|c|c|c|c|c|}
\hline Type & $\begin{array}{c}\left|\delta_{0}\right| \\
10^{-17}\end{array}$ & $\begin{array}{c}E_{\mathrm{LIV}}^{(1)} \\
10^{28} \mathrm{eV}\end{array}$ & $\begin{array}{c}E_{\mathrm{LIV}}^{(2)} \\
10^{21} \mathrm{eV} \\
\end{array}$ & Bound & Source & Reference \\
\hline Limit* & - & 12.08 & 2.38 & $\mathrm{PP}(-)$ & MultiSrc & $\begin{array}{l}\text { Lang, Martínez, } \\
\text { and de Souza (2019) [41] }\end{array}$ \\
\hline Limit* & - & 3.3 & 0.87 & $\mathrm{PP}(-)$ & Mrk501 & H.E.S.S. and FACT (2017) [73] \\
\hline Limit* & - & 2.6 & 0.78 & $\mathrm{PP}(-)$ & Mrk 501 & H.E.S.S. (2019) [28] \\
\hline Limit* & - & 1.9 & 0.31 & $\mathrm{PP}(-)$ & MultiSrc & Biteau and Williams (2015) [7] \\
\hline Limit $^{+}$ & $\sim 0.001$ & $\sim 10^{10}$ & $\sim 10^{7}$ & $\mathrm{PP}(-)$ & UHECR & $\begin{array}{l}\text { Lang, Martínez, } \\
\text { and de Souza (2018) [40] }\end{array}$ \\
\hline Sens.lim. & - & $\sim 1.22$ & $\sim 0.97$ & $\mathrm{PP}(-)$ & - & CTA Consortium (2019) $[53,54]$ \\
\hline Limit* & - & 9.3 & 0.13 & $\Delta t(-)$ & GRB090510 & Vasileiou et al. (2013) [24] \\
\hline Limit* & - & 0.055 & 0.059 & $\Delta t(-)$ & Crab & MAGIC Collaboration (2017) [26] \\
\hline Limit* & - & 0.036 & 0.085 & $\Delta t(-)$ & Mrk 501 & H.E.S.S. (2019) [28] \\
\hline Limit* & - & 0.021 & 0.026 & $\Delta t(-)$ & Mrk 501 & MAGIC Collaboration (2008) [74] \\
\hline Sens.lim. & - & 25 & 0.54 & $\Delta t(-)$ & GRBs & LHAASO Collaboration (2019) [34] \\
\hline Limit* & - & - & 1.4 & AS (-) & Crab (Tibet) & Satunin (2019) [10] \\
\hline Limit* & - & - & 0.97 & AS $(-)$ & Crab (HAWC) & Satunin (2019) [10] \\
\hline Limit* & - & - & 0.21 & AS (-) & Crab (HEGRA) & $\begin{array}{l}\text { Rubtsov, Satunin, } \\
\text { and Sibiryakov (2017) [9] }\end{array}$ \\
\hline Limit* & - & - & 1200 & $3 \gamma(+)$ & eHWC J1825-134 & HAWC Collaboration (2020) [68] \\
\hline Limit* & - & - & 1010 & $3 \gamma(+)$ & eHWC J1907+063 & HAWC Collaboration (2020) [68] \\
\hline Limit* & - & - & 499 & $3 \gamma(+)$ & Crab(HAWC) & HAWC Collaboration (2020) [68] \\
\hline Limit* & - & - & 410 & $3 \gamma(+)$ & Crab (Tibet) & Satunin (2019) [10] \\
\hline Limit* & - & - & 315 & $3 \gamma(+)$ & eHWC J2019+368 & HAWC Collaboration (2020) [68] \\
\hline Limit $* *$ & - & - & 300 & $3 \gamma(+)$ & Crab (HAWC) & Satunin (2019) [10] \\
\hline Limit* & - & - & 130 & $3 \gamma(+)$ & Crab (HEGRA) & $\begin{array}{l}\text { Astapov, Kirpichnikov, } \\
\text { and Satunin (2019) [75] }\end{array}$ \\
\hline Limit* & 1.29 & 2220 & 80 & $\mathrm{PD}(+)$ & MultiSrc & HAWC Collaboration (2020) [68] \\
\hline Limit* & 1.75 & 1390 & 58 & $\mathrm{PD}(+)$ & eHWC J1825-134 & HAWC Collaboration (2020) [68] \\
\hline Limit* & 2.2 & 990 & 47 & $\mathrm{PD}(+)$ & eHWC J1907+063 & HAWC Collaboration (2020) [68] \\
\hline Limit* & 4.52 & 340 & 23 & $\mathrm{PD}(+)$ & Crab(HAWC) & HAWC Collaboration (2020) [68] \\
\hline Limit ** & - & - & 19 & $\mathrm{PD}(+)$ & Crab (Tibet) & Satunin (2019) [10] \\
\hline Limit* & 7.25 & 170 & 14 & $\mathrm{PD}(+)$ & eHWC J2019+368 & HAWC Collaboration (2020) [68] \\
\hline Limit ** & - & - & 14 & $\mathrm{PD}(+)$ & Crab (HAWC) & Satunin (2019) [10] \\
\hline Limit & - & 15 & 2.8 & $\mathrm{PD}(+)$ & Crab (HEGRA) & Martínez and Lorenzana (2017) [8] \\
\hline Limit & - & 1.7 & 0.65 & $\mathrm{PD}(+)$ & $\begin{array}{l}\text { RX J1713.7-3946 } \\
\text { (H.E.S.S.) }\end{array}$ & Martínez and Lorenzana (2017) [8] \\
\hline Limit & $6 \times 10^{5}$ & - & - & $\mathrm{PD}(+)$ & Tevatron & A. Hohensee et al. (2016) [61] \\
\hline Limit* & 40 & - & - & $\mathrm{PD}(+)$ & Crab (HEGRA) & Schreck (2013) [65] \\
\hline Limit & 50 & - & - & $\mathrm{PD}(+)$ & Crab (CANGAROO) & Stecker and Glashow (2001) [49] \\
\hline Limit* & 180 & - & - & $\mathrm{PD}(+)$ & $\begin{array}{l}\text { RX J1713.7-3946 } \\
\text { (H.E.S.S.) }\end{array}$ & Klinkhamer and Schreck (2008) [6] \\
\hline Limit & 300 & - & - & $\mathrm{PD}(+)$ & Crab (Themistocle) & Coleman and Glashow (1997) [13] \\
\hline Sens.lim. & - & $\sim 10^{2}$ & $\sim 10$ & $\mathrm{PD}(+)$ & - & SGSO Alliance $[71,72]$ \\
\hline Limit* & - & 13.4 & 0.09 & $\Delta t(+)$ & GRB090510 & Vasileiou et al. (2013) [24] \\
\hline Limit* & - & 0.026 & 0.073 & $\Delta t(+)$ & Mrk 501 & H.E.S.S. (2019) [28] \\
\hline Limit* & - & 0.045 & 0.053 & $\Delta t(+)$ & Crab & MAGIC Collaboration (2017) [26] \\
\hline Sens.lim. & - & 25 & 0.54 & $\Delta t(+)$ & GRBs & LHAASO Collaboration (2019) [34] \\
\hline
\end{tabular}

${ }^{*}$ Limits that explicitly report having $\sim 95 \%$ (or $2 \sigma$ ) of Confidence Level (C.L.). ${ }^{* *}$ Limits that explicitly report having $5 \sigma$ of C.L. + Limits from the astrophysical scenario which best describes UHECR data. 


\section{Photon Splitting}

Another process forbidden in LI physics which might be allowed if superluminal LIV is considered is the photon splitting, $\gamma \rightarrow \gamma^{\prime}$ s. Here, we discuss the CPT conserving case, $\gamma \rightarrow 3 \gamma$, with $n=2[10,76]$. In contrast with the photon decay, this process does not involve a threshold. Its rate, however, depends on the energy and there is a probability for a photon not splitting on its way to Earth,

$$
P=e^{-L_{\text {source }} /\left\langle L_{\gamma \rightarrow 3 \gamma}\right\rangle},
$$

where $L_{\text {source }}$ is the distance between the source and Earth, and $\left\langle L_{\gamma \rightarrow 3 \gamma}\right\rangle$ is the mean free path for the photon splitting in the quadratic approximation calculated in $[10,75]$, which is given by

$$
\left\langle L_{\gamma \rightarrow 3 \gamma}\right\rangle \approx 16 \mathrm{Mpc}\left(\frac{E_{\mathrm{LIV}}^{(2)}}{10^{14} \mathrm{GeV}}\right)^{10}\left(\frac{E_{\gamma}}{40 \mathrm{TeV}}\right)^{-19} .
$$

By taking the limit case, $L_{\text {source }} /\left\langle L_{\gamma \rightarrow 3 \gamma}\right\rangle=1$, and using the expression above, one can find the energy where the optical depth is 1 ,

$$
E_{\text {cut }} \approx 5.22 \times 10^{11} \mathrm{TeV}\left(\frac{L_{\text {source }}}{\mathrm{kpc}}\right)^{-1 / 19}\left(\frac{E_{\mathrm{LIV}}^{(2)}}{\mathrm{eV}}\right)^{10 / 19} .
$$

The authors of [75] used the absence of such suppression in the Crab Nebula spectrum measured by HEGRA, while the authors of [10] used Tibet to impose limits on the effect. The dedicated exploration for this type of signature on the astrophysical spectra, with energies of gamma rays above $100 \mathrm{TeV}$, made by the HAWC Collaboration, reveals improved exclusion limits through the source eHWC J1825-134 [68]. We also present these limits in Table 1 and Figure 1.

\section{Suppression of Air Shower Formation}

Gamma rays interact with the atmosphere generating a cascade of secondary particles, which are detected by ground-based experiments. This cascade, known as air shower (AS), is stochastic by nature and its first interaction, for the case of gamma rays, is dictated by the Bethe-Heitler process. As first proposed in [77], subluminal LIV may suppress this process causing the AS to be formed deeper in the atmosphere than in the LI case.

The probability for a gamma ray to penetrate the atmosphere up to the atmospheric depth of the experiment, $X_{\text {exp }}$, without initiating an AS and thus not being detected is discussed in [78] and given by

$$
P=1-\exp \left[\left(\frac{X_{\exp }}{57 \mathrm{~g} \mathrm{~cm}^{2}}\right) \frac{12 m_{\mathrm{e}}^{2} E_{\mathrm{LIV}}^{(2)}}{7 E_{\gamma}^{4}} \log \left(\frac{E_{\gamma}^{4}}{2 m_{\mathrm{e}}^{2} E_{\mathrm{LIV}}^{(2)}}\right)\right] .
$$

Similarly to the case to the case of the photon splitting, this would lead to a suppression in the spectrum. The absence of such suppression in the Crab nebula spectrum measured by HEGRA and H.E.S.S. is investigated in [9], while the same is done with the Crab nebula spectrum measured by Tibet in [10], leading to the most restrictive limits so far using this technique, which are also shown in Table 1 and Figure 1.

\section{Final Remarks}

In this work, we have summarized the most common and recent techniques used to explore for signatures of LIV in the photon sector in astrophysical data as well as the latest limits imposed using them, see Table 1 and Figure 1.

In the energy-dependent time delay searches, the main source of uncertainty is the emission time of photons with different energies. The time delay in the emission of photons with different energy 
must be smaller than the time delay caused by LIV propagation. Therefore, a better understanding of the mechanism involved in the emission of photons may lead to a more robust limit. From the experimental perspective, the detection of time delays depends on the detection of flares, only possible with fast slewing telescopes and wide field-of-view satellites.

For the photon pair-production of propagating gamma rays, the main sources of uncertainties are the EBL distribution and the intrinsic spectral shape. The current systematics are discussed in [41]. New EBL measurements are foreseen using the propagation of $\mathrm{TeV}$ photons [79]. The key experimental future is the extension of the detectable energy range to the highest possible energies. Energy spectra with energy above a few $\mathrm{TeV}$ might show a very characteristic and almost background free signal.

Improved limits for both the time delay and the interaction threshold shift are expected within the next years with the construction of the new generation of imaging air-Cherenkov telescopes (IACTs), leaded by the construction of the Cherekov Telescope Array. Better sensitivity and wider energy range will be achieved and, thus, more frequent and energetic GRBs are expected as well as new measurements of $\mathrm{TeV}$ spectra, with more statistics, larger maximum detected energies, and farther sources.

For the UHE photons, on the other hand, the hypothesis about the UHECR source injection, composition and distribution play a determining role as discussed in [40]. In the upcoming years, LIV results will become slightly better due to more restrictive upper limits on the flux coming from longer exposure time. However, the main improvement would come from a better understanding of the UHECR composition which is aimed for the next phase of the present UHECR observatories [80].

On the superluminal cases, limits on the photon splitting are usually less restrictive than those obtained via photon decay. The photon splitting results in a change of the spectrum and its effects could only be seen in a study involving the whole spectrum. The photon decay, on the other hand, is an abrupt effect and just the most energetic photon is enough to impose limits. Moreover, for the photon decay, no astrophysical assumptions about the source or the propagation are made and only the systematics on the energy estimation influence the resulting limits. For this reason, these limits are more robust and less model-dependent. Improvement in these limits depend on the detection of more energetic photons, which may be possible with future experiments such as the SWGO [71,72].

In summary, astrophysics has proven to be a crucial tool in the study of Lorentz invariance violation with stringent limits recently imposed by several works. This has lead to an increase in the interest of the community in this subject and to great expectations for the following years, with the development of new analysis techniques, improvement of data, due to better statistics and better understanding of systematics and new generation of experiments.

Author Contributions: Conceptualization, H.M.-H.; writing-original draft preparation, H.M.-H., R.G.L., and V.d.S.; writing-review and editing, H.M.-H., R.G.L., and V.d.S. All authors have read and agreed to the published version of the manuscript.

Funding: The authors acknowledge FAPESP support No. 2015/15897-1, No. 2016/24943-0, No. 2017/03680-3 and No. 2019/01653-4. The authors also acknowledge the National Laboratory for Scientific Computing (LNCC/MCTI, Brazil) for providing HPC resources of the SDumont supercomputer, which have contributed to the research results reported within this paper (http:/ / sdumont.lncc.br). VdS acknowledges CNPq.

Conflicts of Interest: The authors declare no conflict of interest. The funders had no role in the design of the study; in the collection, analyses, or interpretation of data; in the writing of the manuscript; or in the decision to publish the results.

\section{Abbreviations}

The following abbreviations are used in this manuscript:

LI Lorentz invariance

LIV Lorentz invariance violation

EBL Extragalactic background bight 


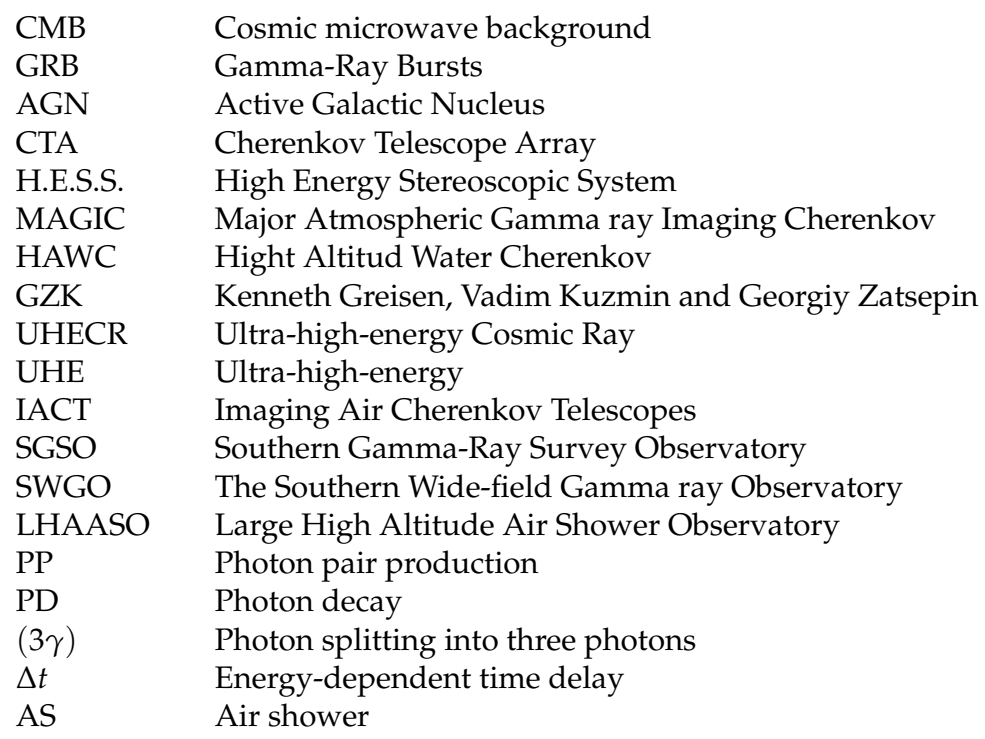

\section{References}

1. Colladay, D.; Kostelecký, V.A. Lorentz violating extension of the standard model. Phys. Rev. 1998, D58, 116002. [CrossRef]

2. Kostelecky, V.A.; Russell, N. Data Tables for Lorentz and CPT Violation. Rev. Mod. Phys. 2011, 83, 11-31. [CrossRef]

3. Alfaro, J. Quantum gravity and Lorentz invariance deformation in the standard model. Phys. Rev. Lett. 2005, 94, 221302. [CrossRef] [PubMed]

4. Bluhm, R. Observational Constraints on Local Lorentz Invariance. In Springer Handbook of Spacetime; Ashtekar, A., Petkov, V., Eds.; Springer: Berlin/Heidelberg, Germany, 2014; pp. 485-507. [CrossRef]

5. Jacobson, T.; Liberati, S.; Mattingly, D. Threshold effects and Planck scale Lorentz violation: Combined constraints from high energy astrophysics. Phys. Rev. D 2003, 67, 124011. [CrossRef]

6. Klinkhamer, F.R.; Schreck, M. New two-sided bound on the isotropic Lorentz-violating parameter of modified-Maxwell theory. Phys. Rev. 2008, D78, 085026. [CrossRef]

7. Biteau, J.; Williams, D.A. The extragalactic background light, the Hubble constant, and anomalies: Conclusions from 20 years of TeV gamma-ray observations. Astrophys. J. 2015, 812, 60. [CrossRef]

8. Martínez-Huerta, H.; Pérez-Lorenzana, A. Restrictions from Lorentz invariance violation on cosmic ray propagation. Phys. Rev. 2017, D95, 063001. [CrossRef]

9. Rubtsov, G.; Satunin, P.; Sibiryakov, S. Constraints on violation of Lorentz invariance from atmospheric showers initiated by multi-TeV photons. JCAP 2017, 1705, 049. [CrossRef]

10. Satunin, P. New constraints on Lorentz Invariance violation from Crab Nebula spectrum beyond $100 \mathrm{TeV}$. Eur. Phys. J. C 2019, 79, 1011. [CrossRef]

11. Martínez-Huerta, H. Lorentz violation constraints with astroparticle physics. In Proceedings of the 8th Meeting on CPT and Lorentz Symmetry (CPT'19) Bloomington, Bloomington, IN, USA, 12-16 May 2019. Available online: http:/ / xxx.lanl.gov/abs/1906.06293 (accessed on 12 May 2020).

12. Nambu, Y. Quantum Electrodynamics in Nonlinear Gauge. Suppl. Prog. Theor. Phys. 1968, 68, $190-195$. [CrossRef]

13. Coleman, S.R.; Glashow, S.L. Cosmic ray and neutrino tests of special relativity. Phys. Lett. 1997, B405, 249-252. [CrossRef]

14. Sarkar, S. Possible astrophysical probes of quantum gravity. Mod. Phys. Lett. 2002, A17, 1025-1036. [CrossRef]

15. Amelino-Camelia, G.; Ellis, J.R.; Mavromatos, N.E.; Nanopoulos, D.V.; Sarkar, S. Tests of quantum gravity from observations of gamma-ray bursts. Nature 1998, 393, 763-765. [CrossRef]

16. Ahluwalia, D.V. Quantum gravity: Testing time for theories. Nature 1999, 398, 199. [CrossRef] 
17. Amelino-Camelia, G. A Phenomenological description of quantum gravity induced space-time noise. Nature 2001, 410, 1065-1067. [CrossRef]

18. Jacob, U.; Piran, T. Lorentz-violation-induced arrival delays of cosmological particles. JCAP 2008, $0801,031$. [CrossRef]

19. Biller, S.D. Limits to quantum gravity effects from observations of TeV flares in active galaxies. Phys. Rev. Lett. 1999, 83, 2108-2111. [CrossRef]

20. Ade, P.A.R.; Aghanim, N.; Arnaud, M.; Ashdown, M.; Aumont, J.; Baccigalupi, C.; Banday, A.J.; Barreiro, R.B.; Bartlett, J.G.; Bartolo, N.; et al. Planck 2015 results. XIII. Cosmological parameters. Astron. Astrophys. 2016, 594, A13. [CrossRef]

21. Ellis, J.R.; Mavromatos, N.E.; Nanopoulos, D.V.; Sakharov, A.S. Quantum-gravity analysis of gamma-ray bursts using wavelets. Astron. Astrophys. 2003, 402, 409-424. [CrossRef]

22. Zou, X.B.; Deng, H.K.; Yin, Z.Y.; Wei, H. Model-Independent Constraints on Lorentz Invariance Violation via the Cosmographic Approach. Phys. Lett. 2018, B776, 284-294. [CrossRef]

23. Zhang, Y.; Liu, X.; Qi, J.; Zhang, H. Cosmological Model Independent Time Delay Method. J. Cosmol. Astropart. Phys. 2018, 2018, 027. [CrossRef]

24. Vasileiou, V.; Jacholkowska, A.; Piron, F.; Bolmont, J.; Couturier, C.; Granot, J.; Stecker, F.W.; Cohen-Tanugi, J.; Longo, F. Constraints on Lorentz Invariance Violation from Fermi-Large Area Telescope Observations of Gamma-Ray Bursts. Phys. Rev. 2013, D87, 122001. [CrossRef]

25. Zitzer, B. Lorentz Invariance Violation Limits from the Crab Pulsar using VERITAS. In Proceedings of the 33rd International Cosmic Ray Conference (ICRC2013), Rio de Janeiro, Brazil, 2-9 July 2013; p. 1147. Available online: http: / xxx.lanl.gov/abs/1307.8382 (accessed on 12 May 2020).

26. Ahnen, M.L.; Ansoldi, S.; Antonelli, L.A.; Arcaro, C.; Babić, A.; Banerjee, B.; Bangale, P.; de Almeida, U.B.; Barrio, J.A.; González, J.B.; et al. Constraining Lorentz invariance violation using the Crab Pulsar emission observed up to TeV energies by MAGIC. Astrophys. J. Suppl. 2017, 232, 9. [CrossRef]

27. Gaug, M.; Garrido, D. Constraining Lorentz invariance violations using the Crab pulsar TeV emission. In Proceedings of the 35th International Cosmic Ray Conference (ICRC2017), Busan, Korea, 10-20 July 2017.

28. Abdalla, H.; Aharonian, F.; Benkhali, F.A.; Angüner, E.O.; Arakawa, M.; Arcaro, C.; Armand, C.; Arrieta, M.; Backes, M.; Barnard, M.; et al. The 2014 TeV $\gamma$-Ray Flare of Mrk 501 Seen with H.E.S.S.: Temporal and Spectral Constraints on Lorentz Invariance Violation. Astrophys. J. 2019, 870, 93. [CrossRef]

29. Ellis, J.R.; Mavromatos, N.E.; Nanopoulos, D.V.; Sakharov, A.S.; Sarkisyan, E.K.G. Robust limits on Lorentz violation from gamma-ray bursts. Astropart. Phys. 2006, 25, 402-411. [CrossRef]

30. Ellis, J.R.; Mavromatos, N.E.; Nanopoulos, D.V.; Sakharov, A.S.; Sarkisyan, E.K.G. Robust limits on Lorentz violation from gamma-ray bursts. Astropart. Phys. 2008, 29, 158-159. [CrossRef]

31. Rodriguez Martinez, M.; Piran, T. Constraining Lorentz violations with gamma-ray bursts. JCAP 2006, 0604, 006. [CrossRef]

32. Perennes, C.; Sol, H.; Bolmont, J. Modeling spectral lags in active galactic nucleus flares in the context of Lorentz invariance violation searches. Astron. Astrophys. 2020, 633, A143. [CrossRef]

33. Pan, Y.; Qi, J.; Cao, S.; Liu, T.; Liu, Y.; Geng, S.; Lian, Y.; Zhu, Z.H. Model-independent constraints on Lorentz invariance violation: Implication from updated Gamma-ray burst observations. Astrophys. J. 2020, 890, 169. [CrossRef]

34. Bai, X. The Large High Altitude Air Shower Observatory (LHAASO) Science White Paper. arXiv 2019. arXiv:1905.02773.

35. De Angelis, A.; Galanti, G.; Roncadelli, M. Transparency of the Universe to gamma rays. Mon. Not. Roy. Astron. Soc. 2013, 432, 3245-3249. [CrossRef]

36. Martínez-Huerta, H.; Pérez-Lorenzana, A. Photon emission and decay from generic Lorentz Invariance Violation. J. Phys. Conf. Ser. 2017, 866, 012006. [CrossRef]

37. Scully, S.T.; Stecker, F.W. Lorentz Invariance Violation and the Observed Spectrum of Ultrahigh Energy Cosmic Rays. Astropart. Phys. 2009, 31, 220-225. [CrossRef]

38. Stecker, F.W.; Scully, S.T. Searching for New Physics with Ultrahigh Energy Cosmic Rays. New J. Phys. 2009, 11, 085003. [CrossRef] 
39. Galaverni, M.; Sigl, G. Lorentz Violation in the Photon Sector and Ultra-High Energy Cosmic Rays. Phys. Rev. Lett. 2008, 100, 021102. [CrossRef] [PubMed]

40. Guedes Lang, R.; Martínez-Huerta, H.; de Souza, V. Limits on the Lorentz Invariance Violation from UHECR astrophysics. Astrophys. J. 2018, 853, 23. [CrossRef]

41. Lang, R.G.; Martínez-Huerta, H.; de Souza, V. Improved limits on Lorentz invariance violation from astrophysical gamma-ray sources. Phys. Rev. 2019, D99, 043015. [CrossRef]

42. Martínez-Huerta, H.; Lang, R.G.; de Souza, V. The optical depth including Lorentz invariance violation energy threshold shifts. arXiv 2018, arXiv:1901.03205.

43. Breit, G.; Wheeler, J.A. Collision of Two Light Quanta. Phys. Rev. 1934, 46, 1087-1091. [CrossRef]

44. Lang, R.G.; Martínez-Huerta, H.; de Souza, V. New stringent LIV limits from astrophysical gamma-ray sources. In Proceedings of the 36th International Cosmic Ray Conference (ICRC2019), Madison, WI, USA, 24 July-1 August 2019; Volume 658. Available online: https:/ / pos.sissa.it/358/658/pdf (accessed on 12 May 2020).

45. Lang, R.G.; Martínez-Huerta, H.; de Souza, V. Competitive subluminal LIV limits from ultra-high energy astrophysics. In Proceedings of the 36th International Cosmic Ray Conference (ICRC2019), Madison, WI, USA, 24 July-1 August 2019; Volume 328. Available online: https:/ / pos.sissa.it/358/328/pdf (accessed on 12 May 2020).

46. Dominguez, A.; Primack, J.R.; Rosario, D.J.; Prada, F.; Gilmore, R.C.; Faber, S.M.; Koo, D.C.; Somerville, R.S.; Pérez-Torres, M.A.; Pérez-González, P.; et al. Extragalactic Background Light Inferred from AEGIS Galaxy SED-type Fractions. Mon. Not. Roy. Astron. Soc. 2011, 410, 2556. [CrossRef]

47. Franceschini, A.; Rodighiero, G.; Vaccari, M. The extragalactic optical-infrared background radiations, their time evolution and the cosmic photon-photon opacity. Astron. Astrophys. 2008, 487, 837. [CrossRef]

48. Gilmore, R.C.; Somerville, R.S.; Primack, J.R.; Dominguez, A. Semi-analytic modeling of the EBL and consequences for extragalactic gamma-ray spectra. Mon. Not. Roy Astron. Soc. 2012, 422, 3189. [CrossRef]

49. Stecker, F.W.; Glashow, S.L. New tests of Lorentz invariance following from observations of the highest energy cosmic gamma-rays. Astropart. Phys. 2001, 16, 97-99. [CrossRef]

50. Stecker, F.W. Constraints on Lorentz invariance violating quantum gravity and large extra dimensions models using high energy gamma-ray observations. Astropart. Phys. 2003, 20, 85-90. [CrossRef]

51. Acharya, B.S. Science with the Cherenkov Telescope Array; WSP: Helsinki, Finland, 2018. Available online: http:/ / xxx.lanl.gov/abs/1709.07997 (accessed on 12 May 2020). [CrossRef]

52. Daniel, M. Lorentz invariance violation with gamma rays. Nucl. Part. Phys. Proc. 2015, 265-266, 314-316. [CrossRef]

53. Gaté, F.; Alves Batista, R.; Biteau, J.; Lefaucheur, J.; Mangano, S.; Meyer, M.; Piel, Q.; Pita, S.; Sanchez, D.; Vovk, I. Studying cosmological $\gamma$-ray propagation with the Cherenkov Telescope Array. arXiv 2018, arXiv:1709.04185. [CrossRef]

54. Martínez-Huerta, H.; Biteau, J.; Lefaucheur, J.; Meyer, M.; Pita, S.; Vovk, I. Testing cosmology and fundamental physics with the Cherenkov Telescope Array. In Proceedings of the 36th International Cosmic Ray Conference (ICRC 2019), Madison, WI, USA, 24 July-1 August 2019. Available online: http:/ / xxx.lanl.gov/abs/1907.08141 (accessed on 12 May 2020).

55. Aab, A.; Abreu, P.; Aglietta, M.; Samarai, I.A.; Albuquerque, I.F.M.; Allekotte, I.; Almela, A.; Castillo, J.A.; Alvarez-Muñiz, J.; Anastasi, G.A.; et al. Search for photons with energies above $10^{18} \mathrm{eV}$ using the hybrid detector of the Pierre Auger Observatory. JCAP 2017, 1704, 009. [CrossRef]

56. Galaverni, M.; Sigl, G. Lorentz Violation and Ultrahigh-Energy Photons. Phys. Rev. 2008, D78, 063003. [CrossRef]

57. Greisen, K. End to the Cosmic-Ray Spectrum? Phys. Rev. Lett. 1966, 16, 748-750. [CrossRef]

58. Zatsepin, G.T.; Kuzmin, V.A. Upper limit of the spectrum of cosmic rays. JETP Lett. 1966, 4, 78-80.

59. Unger, M.; Farrar, G.R.; Anchordoqui, L.A. Origin of the ankle in the ultrahigh energy cosmic ray spectrum, and of the extragalactic protons below it. Phys. Rev. 2015, D92, 123001. [CrossRef]

60. Lang, R.G. Testing Lorentz Invariance Violation at the Pierre Auger Observatory. In Proceedings of the 36th International Cosmic Ray Conference (ICRC2019), Madison, WI, USA, 24 July-1 August 2019; p. 327. Available online: https:/ / pos.sissa.it/358/327/pdf (accessed on 12 May 2020). 
61. Hohensee, M.A.; Lehnert, R.; Phillips, D.F.; Walsworth, R.L. Limits on isotropic Lorentz violation in QED from collider physics. Phys. Rev. 2009, D80, 036010. [CrossRef]

62. Tanimori, T.; Sakurazawa, K.; Dazeley, S.A.; Edwards, P.G.; Hara, T.; Hayami, Y.; Kamei, S.; Kifune, T.; Konishi, T.; Matsubara, Y.; et al. Detection of gamma-rays up to $50-\mathrm{TeV}$ from the Crab Nebula. Astrophys. J. 1998, 492, L33-L36. [CrossRef]

63. Aharonian, F.; Akhperjanian, A.; Beilicke, M.; Bernlöhr, K.; Börst, H.-G.; Bojahr, H.; Bolz, O.; Coarasa, T.; Contreras, J.L.; Cortina, J.; et al. The Crab nebula and pulsar between 500-GeV and 80-TeV. Observations with the HEGRA stereoscopic air Cerenkov telescopes. Astrophys. J. 2004, 614, 897-913. [CrossRef]

64. Aharonian, F. Primary particle acceleration above $100 \mathrm{TeV}$ in the shell-type Supernova Remnant RX J1713.7-3946 with deep H.E.S.S. observations. Astron. Astrophys. 2007, 464, 235-243. [CrossRef]

65. Schreck, M. Obtaining bounds from ultra-high energy cosmic rays in isotropic modified Maxwell theory. In Proceedings of the 6th Meeting on CPT and Lorentz Symmetry (CPT 13), Bloomington, IN, USA, 17-21 June 2013; pp. 176-179. Available online: http:/ /xxx.lanl.gov/abs/1310.5159 (accessed on 12 May 2020). [CrossRef]

66. Abeysekara, A.U.; Albert, A.; Alfaro, R.; Alvarez, C.; Álvarez, J.D.; Camacho, J.R.A.; Arceo, R.; Arteaga-Velázquez, J.C.; Arunbabu, K.P.; Rojas, D.A.; et al. Measurement of the Crab Nebula Spectrum Past $100 \mathrm{TeV}$ with HAWC. Astrophys. J. 2019, 881, 134. [CrossRef]

67. Abeysekara, A.U.; Albert, A.; Alfaro, R.; Camacho, J.R.A.; Arteaga-Velázquez, J.C.; Arunbabu, K.P.; Rojas, D.A.; Solares, H.A.A.; Baghmanyan, V.; Belmont-Moreno, E.; et al. A New Population of Ultra-High-Energy Gamma-Ray Sources Detected by HAWC. 2019. Available online: http://xxx.lanl. gov/abs/1909.08609 (accessed on 12 May 2020).

68. Albert, A.; Alfaro, R.; Alvarez, C.; Camacho, J.R.A.; Arteaga-Velázquez, J.C.; Arunbabu, K.P.; Rojas, D.A.; Solares, H.A.A.; Baghmanyan, V.; Belmont-Moreno, E.; et al. Constraints on Lorentz invariance violation from HAWC observations of gamma rays above $100 \mathrm{TeV}$. Phys. Rev. Lett. 2020, 124, 131101. [CrossRef]

69. Martínez-Huerta, H.; Marinelli, S.; Linnemann, J.T.; Lundeen, J. Constraints on Lorentz invariance violation using HAWC observations above $100 \mathrm{TeV}$. In Proceedings of the HAWC Contributions to the 36th International Cosmic Ray Conference (ICRC2019), Madison, WI, USA, 24 July-1 August 2019. Available online: http:/ /xxx.lanl.gov/abs/1908.09614 (accessed on 12 May 2020).

70. Linnemann, J.T. Lorentz Invariance Violation Limits from HAWC. In Proceedings of the 8th Meeting on CPT and Lorentz Symmetry (CPT'19), Bloomington, IN, USA, 12-16 May 2019.

71. Abreu, P.; Albert, A.; Alfaro, R.; Alvarez, C.; Arceo, R.; Assis, P.; Barao, F.; Bazo, J.; Beacom, J.F.; Bellido, J.; et al. The Southern Wide-Field Gamma-Ray Observatory (SWGO): A Next-Generation Ground-Based Survey Instrument for VHE Gamma-Ray Astronomy. arXiv 2019. arXiv:1907.07737.

72. Albert, A.; Alfaro, R.; Ashkar, H.; Alvarez, C.; Álvarez, J.; Arteaga-Velázquez, J.C.; Solares, H.A.A.; Arceo, R.; Bellido, J.A.; BenZvi, S.; et al. Science Case for a Wide Field-of-View Very-High-Energy Gamma-Ray Observatory in the Southern Hemisphere. arXiv 2019. arXiv:1902.08429.

73. Cologna, G.; Chakraborty, N.; Jacholkowska, A.; Lorentz, M.; Mohamed, M.; Perennes, C.; Romoli, C.; Wagner, S.J.; Wierzcholska, A.; Dorner, D.; et al. The Exceptional Flare of Mrk 501 in 2014: Combined Observations with H.E.S.S. and FACT. AIP Conf. Proc. 2017, 1792, 050019. [CrossRef]

74. Albert, J.; Aliu, E.; Anderhub, H.; Antonelli, L.A.; Antoranz, P.; Backes, M.; Baixeras, C.; Barrio, J.A.; Bartko, H.; Bastieri, D.; et al. Probing Quantum Gravity using Photons from a flare of the active galactic nucleus Markarian 501 Observed by the MAGIC telescope. Phys. Lett. 2008, B668, 253-257. [CrossRef]

75. Astapov, K.; Kirpichnikov, D.; Satunin, P. Photon splitting constraint on Lorentz Invariance Violation from Crab Nebula spectrum. JCAP 2019, 1904, 054. [CrossRef]

76. Gelmini, G.; Nussinov, S.; Yaguna, C.E. On photon splitting in theories with Lorentz invariance violation. JCAP 2005, 0506, 012. [CrossRef]

77. Vankov, H.; Stanev, T. Lorentz invariance violation and the QED formation length. Phys. Lett. B 2002, 538, 251-256. [CrossRef]

78. Rubtsov, G.; Satunin, P.; Sibiryakov, S. On calculation of cross sections in Lorentz violating theories. Phys. Rev. D 2012, 86, 085012. [CrossRef] 
79. Pimentel, D.d.M.; Moura-Santos, E. Infrared emission from dust and the spectral features of extragalactic gamma-ray sources. JCAP 2019, 04, 043. [CrossRef]

80. Aab, A.; Abreu, P.; Aglietta, M.; Ahn, E.J.; Samarai, I.A.; Albuquerque, I.F.M.; Allekotte, I.; Allison, P.; Almela, A.; Castillo, J.A.; et al. The Pierre Auger Observatory Upgrade-Preliminary Design Report. 2016. arXiv:1604.03637. Available online: http:/ / xxx.lanl.gov/abs/1604.03637 (accessed on 12 May 2020).

(C) 2020 by the authors. Licensee MDPI, Basel, Switzerland. This article is an open access article distributed under the terms and conditions of the Creative Commons Attribution (CC BY) license (http:/ / creativecommons.org/licenses/by/4.0/). 